\title{
Heartbeat: Left atrial appendage occlusion for stroke prevention
}

Stroke prevention is the primary goal of therapy in patients with atrial fibrillation (AF). ${ }^{1}$ Heart has published numerous papers on early AF detection, ${ }^{2}$ approaches for restoring normal sinus rhythm, risk scores for determining which AF patients benefit most from anti-thrombotic therapy, and transcatheter occlusion of the left atrial appendage (LAA) to prevent thrombus formation. ${ }^{3-6}$ Early studies of LAA occlusion compared this procedure to vitamin $\mathrm{K}$ antagonist (VKA) therapy for stroke prevention. Now, direct oral anticoagulants (DOACs) have replaced VKA therapy in many AF patients due to an improved risk/benefit profile, among other advantages, but LAA occlusion has not been compared to DOAC therapy.

In this issue of Heart, Sahay and colleagues (see page 139) performed a network meta-analysis of the efficacy and safety of LAA occlusion compared to medical therapy based on 19 randomized trials (including over 87 thousand patients) that compared VKA with placebo, anti-platelet therapy (APT) or a DOAC. Outcomes in 2 major trials of LAA occlusion then were indirectly compared to the network meta-analysis of medical therapy, using VKA therapy as the reference standard (figure 1). The authors conclude that LAA occlusion is more beneficial than placebo or APT and similar in efficacy to DOAC therapy.

In the accompanying editorial, Mazurek and Lip (see page 93) provide a detailed table summarizing the 2 major randomized trials of LAA closure. They also point out that although $90 \%$ of atrial thrombi are located in the LAA, there also is evidence to support the concept that "AF is a systemic disease and thrombogenesis in AF is also multifactorial, including all aspects of Virchow's triad, that is, endothelial or endocardial damage/ dysfunction, abnormal blood stasis (not limited to LAA only) as well as abnormal haemostasis, platelet function and fibrinolysis". Clearly, LAA occlusion will not mitigate this systemic process and also will not prevent stroke related to vascular disease. For those interested in reading

Correspondence to Professor Catherine M Otto, Division of Cardiology, University of Washington, Seattle, WA 98195, USA; cmotto@u.washington.edu further, a recent review article in Heart by Akoum provides an in-depth discussion of the relationships between AF, atrial fibrosis and stoke. ${ }^{7}$ Mazurek and Lip conclude: "For now, oral anticoagulation (whether with an DOAC or warfarin with time in therapeutic range $>70 \%$ ) remains the standard of care therapy for stroke prevention in non-valvular AF. LAA occlusion could possibly be an option, where patients really cannot take any antithrombotic drugs, notwithstanding the fact that some thromboprophylaxis periprocedure and postprocedure for LAA occlusion is often needed. The question remains that if the patient can tolerate aspirin or VKA during this time, some compelling reasons may be needed to justify why they cannot be used longer".
Timely reperfusion with primary percutaneous coronary intervention (PPCI) is the standard of care for acute ST elevation myocardial infarction (STEMI). Varcoe and colleagues (see page 117) hypothesized that the time from the initial patient call to therapeutic intervention of the culprit coronary artery (CTB) provides a better performance measure for a reperfusion service than the time interval after the patient arrives at the medical center, e.g. "door to balloon time" (DTB). Using the British Cardiovascular Intervention Society (BCIS) database of over 16 thousand patients with STEMI treated with PPCI in 2011, the median CTB time was 111 minutes with a DTB time of 41 minutes. Factors that increased the CTB time including out-of-hours call times

Figure 2 Kaplan-Meier plot showing 30-day mortality by call-to-balloon time (with 90 and 150 min as cut-points). 


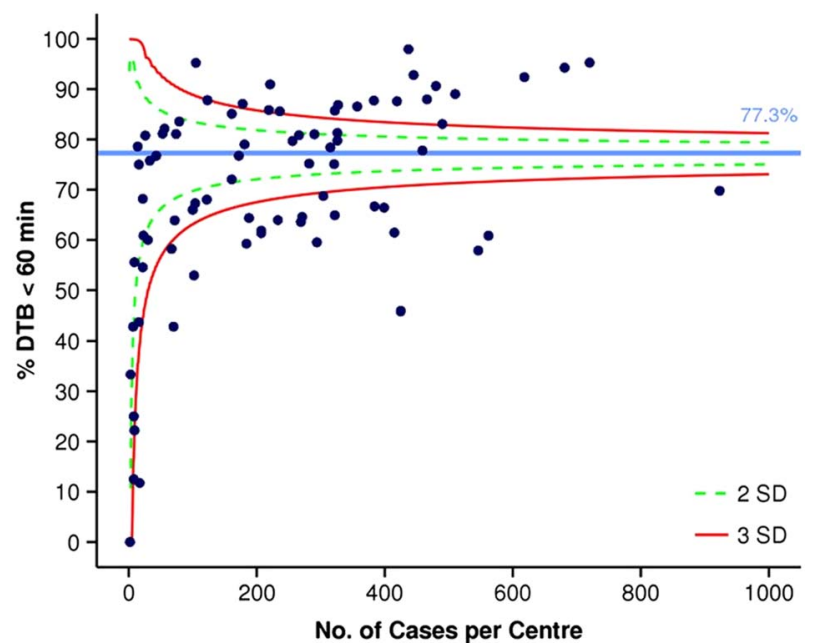

Figure 3 Treatment delays by centre for patients undergoing primary $\mathrm{PCl}(\mathrm{PPCl})$ from the British Cardiovascular Intervention Society (BCIS)/National Institute for Cardiovascular Outcomes Research (NICOR) Audit Report of 2014. Cases include direct admissions and interhospital transfers excluding those requiring preprocedural ventilation and/or cardiogenic shock. Data are shown as funnel plots with 2 and 3 SDs above and below the mean. Proportion of cases with door-to-balloon times $<60$ min. 'Reproduced with permission from the 2014 BCIS Audit Report'.

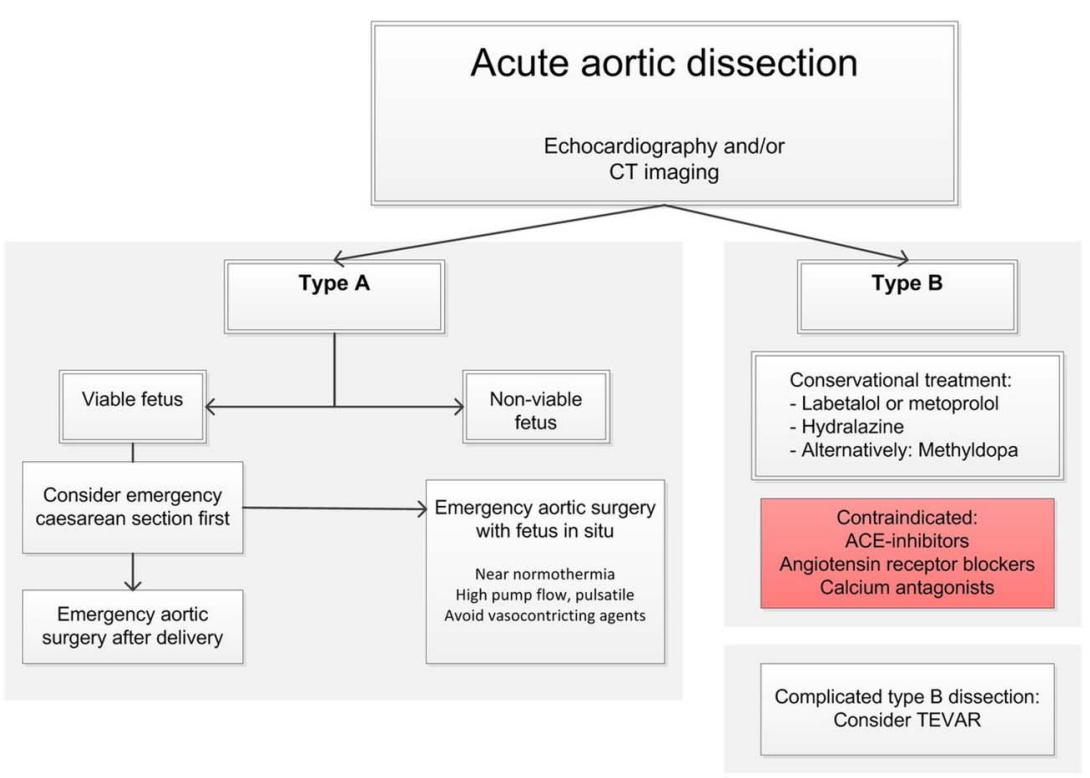

Figure 4 Management of acute aortic dissection during pregnancy.

(by 10 minutes) and inter-hospital transfer (by 49 minutes). A CTB time over 150 minutes was associated with increased 30-day mortality compared to a CTB time of 90 minutes or less (figure 2).

In an editorial entitled "Time is still muscle and there is still room for improvement", Oldroyd (see page 96) provides data from the BCIS Annual Audit Reports on the relationship between centre volume and DTB times. (figure 3) He concludes: "it is essential that individual PPCI centres continuously audit their treatment delays, determine where they stand in relation to the very best practice and implement change when necessary. Generally, the UK is doing well but even here, there remains significant room for improvement".

There are continued advances in coronary artery stent technology. The safety and effectiveness of a biodegradable polymer biolimus-eluting stent (BP-BES) was evaluated in the prospective observational Nobori 2 study (see page 111). In 2738 patients followed for 5 years after BP-BES placement, the composite endpoint of cardiac death, myocardial infarction and target vessel revascularization occurred in $10 \%$ of patients. The strongest predictors of the composite endpoint were previous percutaneous coronary intervention, moderate-to-severe renal disease and peripheral vascular disease. Despite these results, Cassese, Xhepa, and Kastrati (see page 91) suggest that "available data do not support a superior safety of biodegradable-polymer drug-eluting stents (DESs) in comparison with newgeneration durable-polymer DESs". They also point out that "the present report highlights once more that the clinical and angiographic determinants of DES failure remain identical irrespectively of stent platforms."

The Education in Heart article in this issue (see page 159) provides a concise and practical approach for managing cardiac emergencies in pregnancy. Flow charts are provided for management of pregnant women with acute heart failure, supraventricular tachycardia, ventricular tachycardia, acute myocardial infarction, mechanical valve thrombosis, and aortic dissection figure 4. These algorithms might serve as a starting point for more detailed institution-specific checklists and protocols.

The Image Challenge case (see page 153) shows a right atrial mass on cardiovascular MRI as well as histology after resection of the mass. I was surprised by the final diagnosis; perhaps you will be too.

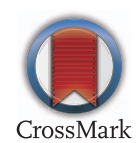

To cite Otto CM. Heart 2017;103:89-90.

Heart 2017:103:89-90.

doi:10.1136/heartjnl-2016-311056

\section{REFERENCES}

1 Jawad-Ul-Qamar M, Kirchhof P. Almanac 2015: atrial fibrillation research in Heart. Heart 2016; 102:573-80.

2 Chan NY, Choy CC. Screening for atrial fibrillation in 13122 Hong Kong citizens with smartphone electrocardiogram. Heart 2017;103:24-31.

3 López Mínguez JR, Asensio JM, Gragera JE. et al. Two-year clinical outcome from the Iberian registry patients after left atrial appendage closure. Heart 2015;101:877-83.

4 Berti S, Pastormerlo LE, Rezzaghi M, et al. Left atrial appendage occlusion in high-risk patients with non-valvular atrial fibrillation. Heart 2016;102:1969-1973.

5 Alli 0 , Holmes D Jr. Left atrial appendage occlusion. Heart 2015;101:834-41.

6 Cabrera JA, Saremi F, Sánchez-Quintana D. Left atrial appendage: anatomy and imaging landmarks pertinent to percutaneous transcatheter occlusion. Heart 2014; 100:1636-50.

7 Akoum N. New perspectives on atrial fibrillation and stroke. Heart 2016;102:1788-92. 